\title{
Oscillatory Pollen Tube Growth: Imaging the Underlying Structures and Physiological Processes
}

\author{
P.K. Hepler,* M. Bosch, ${ }^{*}$ L. Cardenas, ${ }^{*}$ A. Lovy-Wheeler,* S.T. McKenna***, K.L. Wilsen, ${ }^{*}$ and J.G. \\ Kunkel* \\ *Biology Department, University of Massachusetts, Amherst MA 01003 \\ **Institute of Plant Biotechnology, UNAM, Cuernavaca, Morelos 62271, Mexico \\ ***Department of Biology, Long Island University, Brooklyn, NY 11201
}

Pollen tube growth is fast ( $\sim 300 \mathrm{~nm} / \mathrm{sec}$ in lily), highly polarized (tip growth), and oscillatory (100 to 500 $\mathrm{nm} / \mathrm{sec}$ over a period of 20-50 sec)[1]. Many underlying physiological processes and structures also oscillate, always with the same period as growth, but not with the same phase. Through phase analysis we can distinguish between those processes that follow vs. those that anticipate growth (Fig. Graph). The latter gain importance in our quest to identify the core regulator of growth [1]. Here we report on the oscillation and phase relationships of three physiological factors $\left(\mathrm{Ca}^{2+}, \mathrm{pH}, \mathrm{NAD}(\mathrm{P}) \mathrm{H}\right)$, and three structural components (ER, apical cell wall, actin).

$\mathrm{Ca}^{2+}$ : Pollen tubes, injected with fura-2-dextran, possess a tip-focused $\mathrm{Ca}^{2+}$ gradient that oscillates (Fig. $\mathrm{Ca}$ ). Cross-correlation analysis reveals that the $\mathrm{Ca}^{2+}$ peaks $10-30^{0}$ after the growth rate [2]. These data suggest that changes $\mathrm{Ca}^{2+}$ do not serve a primary regulatory role; rather growth regulates $\mathrm{Ca}^{2+}[1,2]$

pH: Pollen tubes, injected with BCECF-dextran, possess an oscillatory pH gradient (6.8 at the tip; 7.5 in the clear zone)[1]. The acidic tip follows growth by $\sim 20^{\circ}$, whereas the alkaline band anticipates growth rate by $\sim 125^{\circ}$. These results point to the alkaline band as a central player in the control of growth.

$\mathrm{NAD}(\mathrm{P}) \mathrm{H}$ : Bound $\mathrm{NAD}(\mathrm{P}) \mathrm{H}$, (endogenous fluorescence) oscillates, with the peaks following growth. However the troughs, (free $\mathrm{NAD}(\mathrm{P}) \mathrm{H}$ ?), anticipate growth by $45^{\circ}$. In addition the troughs show a stronger correlation with growth than do the peaks. Free NAD(P)H may also be key regulator of growth.

ER: ER, stained with mGFP5-ER (Fig. ER)[3], exhibits an oscillatory motion, in which elements move forward along the flanks of the tube and then curl inward, into the apex interior. The inward curling anticipates growth by $\sim 95^{\circ}$. These observations direct attention to actin, since those filaments move ER.

Apical Wall Thickness: DIC reveals that the apical cell wall oscillates in thickness, and precedes growth by $\sim 100^{\circ}$. Parallel studies on endocytosis (Fig. FM4-64) indicate that it follows growth. Exocytosis is probed with pectin methyl esterase-GFP (Fig. PME)[4]. Analysis of phase is still in progress.

Actin: Pollen tubes, which have been fixed by rapid freezing and stained immunofluorescently, reveal a cortical apical fringe of actin (Fig. Actin)[5]. Among the live cell markers, e.g., GFP-ADF and GFP-talin weakly stain the fringe, but GFP-fimbrin does not; studies of F-actin oscillation have not been possible. However, G-actin, stained with fluorescent DNAse, oscillates and follows growth by $\sim 20^{\circ}$.

\section{References:}

[1] Holdaway-Clarke TL, Hepler PK. New Phytol. 159 (2003) 539.

[2] Messerli MA, et al. Dev. Biol. 222 (2000) 84.

[3] Napier RM, et al. J. Cell Sci. 102 (1992) 261.

[4] Bosch M, et al. Plant Physiol. (2005)(submitted).

[5] Lovy-Wheeler A, et al. Planta (2005)(in press).

[6] Research supported by a grant from the NSF (MCB-0077599). 


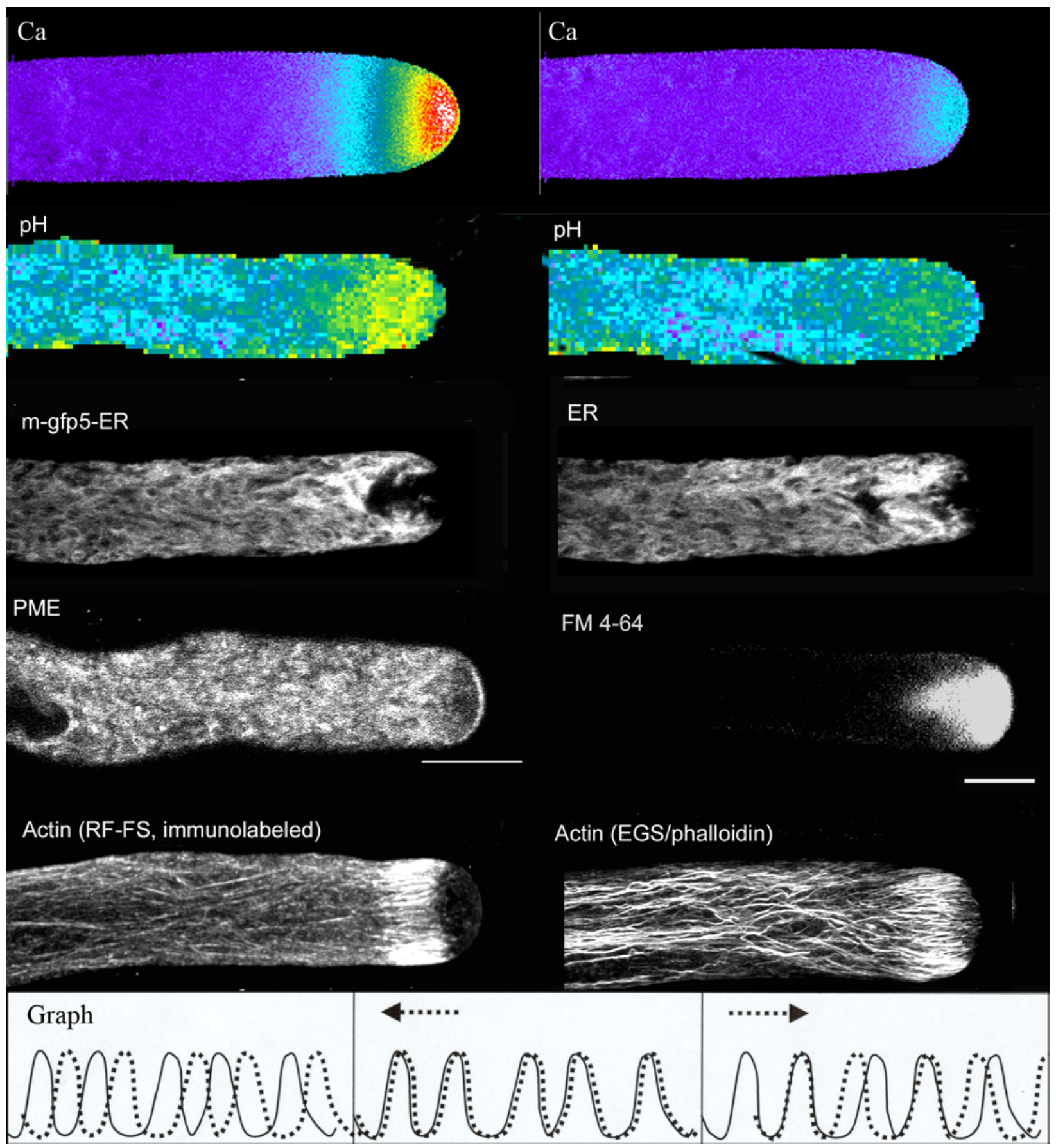

Figure Legend:

Row-1: $\mathrm{Ca}^{2+}$; high (left), low (right). Cell injected with fura-2-dextran (FM4-64 scale bar; $\left.10 \mu \mathrm{m}\right)$. Row-2: pH; high (left, low (right). Cell injected with BCECF-dextran (FM4-64 scale bar; $10 \mu \mathrm{m}$ ). Row-3: ER; stained with mGFP5-ER. Images show different positions of ER (FM4-64 scale bar; $10 \mu \mathrm{m}$ ). Row-4: GFP-PME, exocytosis (left)(Bar; $10 \mu \mathrm{m})$; FM4-64, endocytosis (right)(Bar; $10 \mu \mathrm{m})$.

Row-5: Actin, freeze fix, antibody stain (left); EGS fix, phalloidin stain (right)(FM4-64 scale bar; $10 \mu \mathrm{m}$ ). Row-6: Graph showing cross-correlation. Raw data (left); Shift left (center); Shift right (right). 